\title{
Defining the Lines: Coding for the Neonatal Delivery and Admission
}

Kate Peterson Stanley, MD, FAAP

"As a race, the initial care of a premature
newborn infant begins with the delivery
and ends with the admission. In between,
multiple procedures may be performed
to stabilize the infant. Coding for these
procedures depends on where the
delivery ends and the admission begins."

While watching the Tokyo Olympic Track and Field events this past month, I couldn't help but think about the well-defined beginning and end of each race. Glory or defeat is defined by the runner's performance between the start and finish line. For any given race, the outcome changes depending on what occurs between those two lines. As a race, the initial care of a premature newborn infant begins with the delivery and ends with the admission. In between, multiple procedures may be performed to stabilize the infant. Coding for these procedures depends on where the delivery ends and the admission begins. When procedures are required to support acute inadequate ventilation or cardiac output during delivery room resuscitation, they are coded separately from the delivery room care. In contrast, when procedures are part of ongoing care post-resuscitation, they are bundled with the neonatal critical/intensive care admission codes as a "package" and are not coded separately. Examples include intubation for surfactant administration or umbilical line placement for vital sign monitoring and medication administration. In the past, many providers determined the difference between these two events by location. Procedures performed in the corner of the mother's room to stabilize the infant directly after birth were part of the "delivery room resuscitation." Afterward, the infant was placed in an incubator and wheeled to a bed space in the NICU. Additional procedures were included in the "admission." With the development of Golden Hour protocols and high-tech delivery room resuscitation bays, the lines defining the end of the delivery and the beginning of the NICU admission may be gray. But, despite a change in where we deliver care, the "lines" of CPT coding rules remain the same.

\section{Scenario:}

A neonatologist at a regional neonatal center is called to the ER for an emergency delivery of a 29-week premature infant. The mother is en route to the hospital by ambulance after going into labor while at the shopping mall. The infant is delivered before you arrive. The ER physician provides the initial steps of newborn resuscitation (NRP) at a warmer in the trauma room. When you arrive at the warmer 3 minutes after delivery, you assess the infant, provide PPV, intubate the baby due to persistent respiratory failure and continue to follow NRP guidelines. The infant responds to these resuscitative measures. Apgars are 2/6/8. Birthweight is 1100 grams. You speak with the mother and continue to care for the infant while the NICU prepares a bed, and the ER team attends to another emergency. CXR shows RDS. While waiting, you give surfactant, stabilize the infant on a ventilator, place a UVC and begin starter TPN. When the bed is ready, the infant is transferred to the NICU for further care. The total time you spent in the ER was 65 minutes.

\section{The correct CPT codes for the neonatologist are:}

A. 99465 Delivery room resuscitation

B. 99464 Delivery room attendance

C. 99468 Admission of a critically ill newborn, $<29$ days

D. 99291 Evaluation and management of a critically ill patient, first 30-74 minutes

E. 31500 Intubation

F. 36510 Catheterization of umbilical vein (UVC)

G. 94610 Surfactant

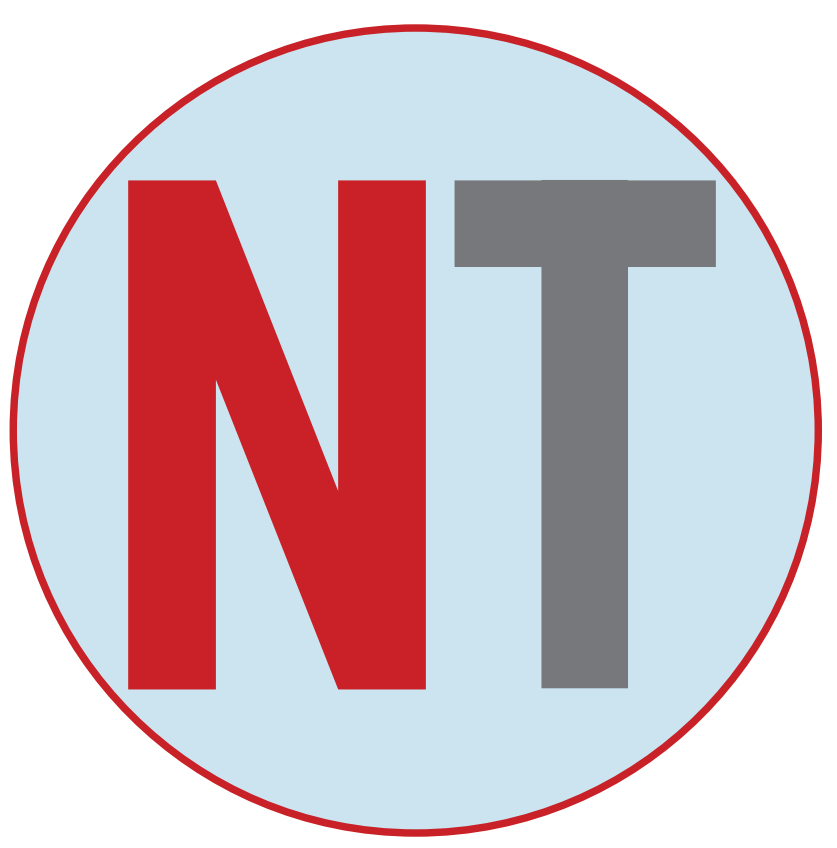

Answer: A, C, E

A. 99465: This CPT code is the correct code for the "work" provided at the newborn's birth resuscitation: providing NRP including PPV and/or chest compressions to establish ventilation and/or cardiac output, visually inspecting the infant, assigning Apgars, and updating the parents. Although the ER physician was the first provider at the resuscitation, the neonatologist provided the majority of the work, so it is appropriate for the neonatologist to bill for the encounter. This code may only be used for resuscitation provided at the time of birth, regardless of birth location. 
B. 99464: This CPT code should be used when resuscitation of the newborn does not require PPV or other measures such as CPR. It includes providing the initial stabilization steps of NRP (drying, stimulation, suctioning), providing blow-by oxygen, CPAP without PPV, visual inspection of the infant, Apgar assignment, and discussion with the parents. Like 99465 , it may only be used at the time of birth, regardless of birth location.

C. 99468: This is the correct CPT code for the inpatient admission of a critically ill newborn $<29$ days of age. It is an agebased, global, per diem, bundled code. The infant meets the criteria for critical care secondary to respiratory failure requiring ventilator management. Critical care may be provided in any location such that the patient has an illness or injury that acutely impacts one or more vital organ systems where there is a high probability of imminent or life-threatening deterioration in the patient's condition. The care provided must involve high complexity decision-making to assess, manipulate and support vital system function to treat vital organ system failure and/or prevent further life-threatening deterioration. In this specific situation, the modifier 25 is appended to the admission code to signify a significant, separately identifiable $\mathrm{E} / \mathrm{M}$ by the same physician on the same day as another service (the delivery room intubation).

D. 99291: This code is used when providing critical care to patients older than 6 years of age, co-managing a critically ill patient in a consultative role or when providing critical care services to a patient transferred to a separate institution on the same day. In the scenario above, the ER physician could have used this code if he/she had assisted the neonatologist with stabilizing the infant after the delivery resuscitation for a minimum of 30 minutes.

E. 31500: The neonatologist may code for the intubation performed during the delivery resuscitation because it is a distinct, separate procedure and was required to support inadequate ventilation immediately following birth. It is not included or "bundled" as part of the resuscitation code. If the neonatologist performed the intubation after the delivery room resuscitation, it is considered part of the admission. The neonatologist would not code for the procedure because it is bundled into the global newborn admission code.

F. 36510: Since the UVC was placed to provide nutritional support as part of the NICU admission, the neonatologist would not code for it. An umbilical line (venous or arterial) is a bundled procedure when using the global newborn admission code 99468. If a UVC was required during the delivery room resuscitation for management of acute inadequate cardiac output, the neonatologist may code for the procedure. If more than one procedure is required during the resuscitation, modifier 59 "Distinct Procedural Service" is added to the additional procedures.

G. 94610: Similar to UVC placement, the neonatologist may not code for surfactant administration because it is bundled with the admission code 99468. On rare occasions, the surfactant is administered as part of newborn resuscitation in acute inadequate ventilation and/or cardiac output. The provision of surfactant immediately following resuscitation as part of a delivery room protocol is considered part of the admission process and is therefore bundled into the global newborn admission code. If the provider is coding for hourly critical care services as a consultant or when transferring the infant to another service or institution for ongoing care, surfactant administration, UVC and intubation may be coded in addition to hourly critical care since they are not bundled procedures when providing this service.

The correct ICD-10 codes are:

Z38.00 Single liveborn infant delivered vaginally

P07.14 Other low birth weight newborn, 1000-1249 grams

P07.32 Preterm newborn, gestational age 29 weeks

P22.0 Respiratory distress syndrome of the newborn

\section{The Finish Line:}

Defining when newborn resuscitation ends and the NICU admission begins is not always easy because each newborn runs a different race. When the lines are gray, I consider this coding pearl: Any procedure required to establish ventilation and cardiac output during newborn resuscitation to obtain an Apgar $>7$ is considered delivery room care and is not included in the admission. With this in mind, I can see the finish line and complete the race.

\section{References:}

1. Duncan S, Martin G, Pearlman S, eds. Quick Reference Guide to Neonatal Coding and Documentation. $2^{\text {nd }}$. American Academy of Pediatrics. 2016.

2. COCN American Academy of Pediatrics. Coding for Pediatrics 2021. American Academy of Pediatrics. 2021.

3. ICD-10-CM 2021, The Complete Official Codebook. American Medical Association. 2021.

Disclosure: The author has no disclosures.

$$
\text { NT }
$$

Corresponding Author:
Kate Peterson Stanley, MD
Medical Director of Revenue Integrity
CS Mott Children's and Von Voigtlander Women's Hospitals
Clinical Assistant Professor
Division of Neonatal-Perinatal Medicine
Department of Pediatrics
University of Michigan Medical School
Mailing Address: 8-621 CS Mott
1540 E. Hospital Dr., SPC4254
Ann Arbor, MI 48109-4254
Phone: 734-763-4109
Fax: 734-763-7728
Email: katest@med.umich.edu

